\title{
CROSS RATIO COORDINATES FOR THE DEFORMATION SPACES OF A MARKED MÖBIUS GROUP
}

\author{
KIMIYO N. YAMAMOTO AND MASAHIKO TANIGUCHI
}

\begin{abstract}
We introduce a new kind of coordinate systems for the deformation space of a finitely generated free Möbius group by using cross ratio functions induced by the fixed points of Möbius transformations. As an application, we give a new complete distance on the Schottky space by using such functions, which is not greater than the Teichmüller distance.
\end{abstract}

\section{INTRODUCTION}

Let $G$ be a free group generated by $n$ Möbius transformations, which we equip with an ordered set $\left(g_{1}, \cdots, g_{n}\right)$ of generators. Here we assume that $n \geq 2$. The rough deformation space $\operatorname{RDef}(G)$ of such a group $G$ is the space of all homomorphisms of $G$ into the space $\mathrm{PSL}_{2}(\mathbb{C})$ of all Möbius transformations. The natural identification between $\operatorname{RDef}(G)$ and $\mathrm{PSL}_{2}(\mathbb{C})^{n}$ is obtained by setting

$$
\sigma \mapsto\left(\sigma\left(g_{1}\right), \cdots, \sigma\left(g_{m}\right)\right)
$$

We say that $\sigma$ and $\sigma^{\prime}$ in $\operatorname{RDef}(G)$ are Möbius conjugate if there is an element $M \in \mathrm{PSL}_{2}(\mathbb{C})$ such that

$$
\sigma^{\prime}(g)=M \sigma(g) M^{-1}
$$

for every $g \in G$. The space of all Möbius conjugacy classes of elements in $\operatorname{RDef}(G)$ is called the deformation space of $G$ and denoted by $\operatorname{Def}(G)$. For every $\sigma \in \operatorname{RDef}(G)$, $[\sigma]$ denotes the point in $\operatorname{Def}(G)$ containing $\sigma$. The natural projection of $\operatorname{RDef}(G)$ to $\operatorname{Def}(G)$ is denoted by $\pi$. Here, there is another quotient space $\operatorname{RDef}(G) / / \mathrm{PSL}_{2}(\mathbb{C})$ in the sense of the invariant theory, which is called the deformation variety $X(G)$ for $G$ in $\mathrm{PSL}_{2}(\mathbb{C})$. It is known that $X(G)$ is an affine algebraic set.

Remark 1.1. Standard parameters on $X(G)$ are trace functions. For a $\sigma \in \operatorname{RDef}(G)$, the character of $\sigma$ is the map

$$
\chi_{\sigma}: G \rightarrow \mathbb{C}, \quad g \in G \rightarrow \operatorname{tr}^{2}(\sigma(g)) .
$$

Since there is a natural bijection between $X(G)$ and the sets of such characters, $X(G)$ is also called the character variety for $G$. But trace functions do not give a system of coordinates for $X(G)$ in general. See, for instance, [4.

Received by the editors December 27, 2012.

2010 Mathematics Subject Classification. Primary 30F40; Secondly 30F45, 30F60.

Key words and phrases. Kleinian group, Schottky spaces, cross ratio.

The first author was supported by JSPS Research Fellowship for Young Scientests (PD) (No. 6811).

The second author was partially supported by Grant-in-Aid for Scientific Research (C) (Grant No. 23540202) and (B) (Grant No. 25287021). 
It is a fundamental problem to give useful systems of coordinates, or parameters, on such deformation spaces as above. To discuss this issue, in the sequel we consider only such $\sigma$ that $\sigma(G)$ is non-abelian and $\sigma\left(g_{k}\right)$ is not the identical transformation for every $k$. The subset $\operatorname{EL}(G)$ of $X(G)$ consisting of all conjugacy classes $[\sigma]$ of $\sigma$ satisfying these conditions is generic and can be considered as a subset of $\operatorname{Def}(G)$. Here we say that a subset $Y$ of an algebraic variety $X$ is generic if $X-Y$ is contained in a finite union of proper algebraic sub-varieties.

Definition 1.2. We call $\operatorname{EL}(G)$ the essential locus.

It is well known that $\operatorname{EL}(G)$ has a standard complex manifold structure. Actually, the rough essential locus $\operatorname{REL}(G)=\pi^{-1}(\operatorname{EL}(G)) \subset \operatorname{RDef}(G)$ is the total space of a principal complex-analytic bundle over $\operatorname{EL}(G)$ with $\pi$ as the projection. (See, for instance, [12.)

Recall that the deformation space $\operatorname{Def}(G)$ contains several important sub-loci other than the essential locus $\operatorname{EL}(G)$.

Definition 1.3. The sub-locus $F(G)$ consisting of all conjugacy classes of isomorphisms is called the faithfulness locus. The sub-locus $D(G)$ consisting of all $[\sigma]$ with discrete $\sigma(G)$ is called the discreteness locus.

The Schottky space $S(G)$, by definition, consists of all $[\sigma]$ such that $\sigma(G)$ are Schottky groups with $n$ generators.

These sub-loci have been considered by many authors from various points of view, and many kinds of useful systems of coordinates have been considered. Also, it is clear that $F(D) \subset \operatorname{EL}(G)$. Further, we have the following strict inclusion relations. (Cf. Proposition 3.1 below.)

$$
S(G) \subsetneq F(G) \cap D(G) \subsetneq \mathrm{EL}(G) .
$$

Remark 1.4. For various kinds of systems of classical coordinates for these deformation spaces, see, for instance, [1], 7], and [8]. Also cf. [15], and [5].

In this paper, we equip $\operatorname{EL}(G)$ with a natural marking induced by order of fixed points of generators, which gives a finite branched holomorphic cover $\widehat{\operatorname{EL}}(G)$ of $\operatorname{EL}(G)$, and we introduce a new kind of the global coordinate systems for generic sets of $\widehat{\mathrm{EL}}(G)$ by considering cross ratios of the fixed points considered as functions on $\widehat{\mathrm{EL}}(G)$. More precisely, see Theorem 2.4 .

Also as an application of such cross ratio functions, we give a new distance on the Schottky space $S(G)$, which is complete and not greater than the Teichmüller distance (Theorem 3.7).

\section{Coordinates induced By CROss Ratios}

Schottky groups are marked by ordered pairs of disjoint non-nested closed topological discs. But such geometric markings are not so easy to generalize to general Möbius groups, and here we use different non-geometric marking as follows.

Definition 2.1. For every point $\sigma \in \operatorname{REL}(G)$, we say that $\sigma$ is marked if every generator $\sigma\left(g_{j}\right)$ is decorated with a point $\mathbf{p}_{j}(\sigma)=\left(p_{2 j-1}, p_{2 j}\right) \in \widehat{\mathbb{C}}^{2}$ such that $\left\{p_{2 j-1}, p_{2 j}\right\}$ is the set Fix $\sigma\left(g_{j}\right)$ of all fixed points of $\sigma\left(g_{j}\right)$. We write $\sigma \in \operatorname{REL}(G)$ with the marking $\mathbf{p}=\mathbf{p}(\sigma)=\left(\mathbf{p}_{1}(\sigma), \cdots, \mathbf{p}_{n}(\sigma)\right) \in \widehat{\mathbb{C}}^{2 n}$ as $\widehat{\sigma}=(\sigma, \mathbf{p})$. The set of 
all ordered pairs $\widehat{\sigma}=(\sigma, \mathbf{p})$ with $\sigma \in \operatorname{REL}(G)$ and possible marking $\mathbf{p}=\mathbf{p}(\sigma)$ is called the rough marked essential locus, and denoted by $\widehat{\operatorname{REL}}(G)$.

We say that $\left(\sigma_{1}, \mathbf{p}_{1}\right)$ and $\left(\sigma_{2}, \mathbf{p}_{2}\right)$ in $\widehat{\operatorname{REL}}(G)$ are Möbius equivalent if there is an $M \in \mathrm{PSL}_{2}(\mathbb{C})$ such that $\mathbf{p}_{2}=M \mathbf{p}_{1}$ and $\sigma_{2}(g)=M \sigma_{1}(g) M^{-1}$ for every $g \in G$. Here, $M\left(p_{1}, \cdots, p_{2 n}\right)=\left(M\left(p_{1}\right), \cdots, M\left(p_{2 n}\right)\right)$.

Let $\widehat{\operatorname{EL}}(G)$ be the set of all Möbius equivalence classes $[\widehat{\sigma}]$ of $\widehat{\sigma} \in \widehat{\operatorname{REL}}(G)$. We call $\widehat{\mathrm{EL}}(G)$ the marked essential locus.

The following fact is clear from the definition.

Proposition 2.2. The marked essential locus $\widehat{\mathrm{EL}}(G)$ has a natural complex structure such that the projection

$$
\tau: \widehat{\operatorname{EL}}(G) \rightarrow \operatorname{EL}(G)
$$

obtained by forgetting the marking gives a $2^{n}$-sheeted holomorphic branched cover.

Now, we introduce a system of global coordinates on a generic set of $\widehat{\operatorname{EL}}(G)$ by using cross ratios. Write the marking of $\widehat{\sigma}$ in $\widehat{\operatorname{REL}}(G)$ as

$$
\mathbf{p}=\left(p_{1}, \cdots, p_{2 n}\right) \text {. }
$$

Then for every $j=1, \cdots, n$ and $k=0,1$, we can consider the marked orbit $M O\left(p_{2 j-k}\right)$ of $p_{2 j-k}$ which consists of the value $p_{2 j-k}$ marked with the identity and the values $\sigma(g)\left(p_{2 j-k}\right)$ marked with elements $g$ of $G$, where $g$ moves over all elements of $G$ whose reduced word representations $g=g_{i_{s}}^{\epsilon_{n}} \cdots g_{i_{1}}^{\epsilon_{1}}\left(s>0, \epsilon_{\nu}= \pm 1\right)$ satisfy the condition that $g_{i_{1}} \neq g_{j}$. Also, we fix an order of elements in $M O\left(p_{m}\right)$ for every $m=1, \cdots, 2 n$, and consider $M O\left(p_{m}\right)$ as an infinite-dimensional vector. Set

$$
\operatorname{ES}(\widehat{\sigma})=\left(M O\left(p_{1}\right), \cdots, M O\left(p_{2 n}\right)\right) \in\left(\widehat{\mathbb{C}}^{\infty}\right)^{2 n},
$$

and define a continuous map

$$
\Sigma_{F P}: \widehat{\operatorname{REL}}(G) \rightarrow\left(\widehat{\mathbb{C}}^{\infty}\right)^{2 n}
$$

by sending $\widehat{\sigma}$ to $\operatorname{ES}(\widehat{\sigma})$. We call the image $\Sigma_{F P}(\widehat{\sigma})=\operatorname{ES}(\widehat{\sigma})$ the rough essential fixed point spectrum of $\widehat{\sigma}$.

Recall that the components $\sigma(g)\left(p_{2 j-1}\right)$ and $\sigma(g)\left(p_{2 j}\right)$ of $\operatorname{ES}(\widehat{\sigma})$ are the fixed points of the element $\sigma\left(g g_{j} g^{-1}\right)$ for every $j$ and $g \in G$ as above.

Definition 2.3. The cross ratio

$$
\chi\left(q_{1}, q_{2}, q_{3}, q_{4}\right)=\frac{q_{1}-q_{2}}{q_{1}-q_{3}} \frac{q_{4}-q_{3}}{q_{4}-q_{2}} \quad\left(\text { if } q_{j} \in \mathbb{C}\right)
$$

of given components $q_{k}$ of $\Sigma_{F P}(\widehat{\sigma})$ is well defined for the conjugacy class $[\widehat{\sigma}]$ and, furthermore, can be considered as a holomorphic function $\widehat{\chi}$ on a generic set $\Omega_{\widehat{\chi}}$ of $\widehat{\mathrm{EL}}(G)$ where no subsets consisting of three corresponding components are coincident to each other. We call such a holomorphic function

$$
\widehat{\chi}: \Omega_{\widehat{\chi}} \rightarrow \widehat{\mathbb{C}}
$$

a FPCR (fixed point cross ratio) function.

We can show that the set of all FPCR functions separates points of $\widehat{\operatorname{EL}}(G)$ in the sense that, for any pair of points in $\widehat{\operatorname{EL}}(G)$, there is a FPCR function defined at both points which takes distinct values at these points. Furthermore, we can introduce a system of global coordinates for a generic subset of $\widehat{\mathrm{EL}}(G)$. 
Theorem 2.4. There is an open cover $\left\{\Omega_{j}\right\}$ of $\widehat{\mathrm{EL}}(G)$ by a finite number of generic subsets such that, for every $\Omega_{j}$, there are $(3 n-3)$ FPCR functions $\left\{\widehat{\chi}_{1}, \cdots, \widehat{\chi}_{3 n-3}\right\}$ defined on $\Omega_{j}$ which gives a holomorphic injection

$$
\Lambda_{\Omega_{j}}=\left(\widehat{\chi}_{1}, \cdots, \widehat{\chi}_{3 n-3}\right): \Omega_{j} \rightarrow \widehat{\mathbb{C}}^{3 n-3} .
$$

Proof. First note that, for every $[\widehat{\sigma}] \in \widehat{\operatorname{EL}}(G)$, there is a pair $\{i, j\}$ with $i \neq j$ such that $\operatorname{Fix}\left(\sigma\left(g_{i}\right)\right) \neq \operatorname{Fix}\left(\sigma\left(g_{j}\right)\right)$. Set

$$
\Omega(\{i, j\})=\left\{[\widehat{\sigma}] \in \widehat{\operatorname{EL}}(G) \mid \operatorname{Fix} \sigma\left(g_{i}\right) \neq \operatorname{Fix} \sigma\left(g_{j}\right)\right\}
$$

and

$$
\Omega(i, j, k)=\left\{[\widehat{\sigma}] \in \widehat{\operatorname{EL}}(G) \mid p_{2 j-k} \notin \operatorname{Fix} \sigma\left(g_{i}\right)\right\} \quad \text { if } k=0,1 .
$$

Then, clearly,

$$
\bigcup_{k=0}^{1}(\Omega(i, j, k) \cup \Omega(j, i, k)) \supset \Omega(\{i, j\}),
$$

and we have a finite open cover $\{\Omega(i, j, k) \mid i \neq j, k=0,1\}$ of $\widehat{\operatorname{EL}}(G)$.

Fix $i, j, k$ with $i \neq j, k=0,1$. Set

$$
A_{i, j, k}=\left\{p_{2 i-1}, p_{2 j-k}, \sigma\left(g_{i}\right)\left(p_{2 j-k}\right)\right\} .
$$

Fix $\left(r_{1}, \cdots, r_{n}\right) \in\left(A_{i, j, k}\right)^{n}$ arbitrarily and abbreviate it as $\mathbf{r}$. Set

$$
\Omega(i, j, k)_{\mathbf{r}}=\left\{[\widehat{\sigma}] \in \Omega(i, j, k) \mid r_{\ell} \neq \sigma\left(g_{\ell}\right)\left(r_{\ell}\right) \text { for every } \ell\right\} .
$$

Then the family

$$
\left\{\Omega(i, j, k)_{\mathbf{r}} \mid i \neq j, k=0,1 ; \mathbf{r} \in\left(A_{i, j, k}\right)^{n}\right\}
$$

gives an open cover of $\widehat{\operatorname{EL}}(G)$. Indeed, every $[\widehat{\sigma}]$ in $\widehat{\operatorname{EL}}(G)$ belongs to some $\Omega(i, j, k)$, and then $A_{i, j, k}$ consists of 3 distinct points. Hence, for every $\ell \neq i$, there is a point $r_{\ell} \in A_{i, j, k}$ such that $r_{\ell} \neq \sigma\left(g_{\ell}\right)\left(r_{\ell}\right)$. Set $r_{i}=p_{2 j-k}$ and take this $\mathbf{r}=\left(r_{1}, \cdots, r_{n}\right) \in$ $\left(A_{i, j, k}\right)^{n}$. Then $[\widehat{\sigma}]$ belongs to $\Omega(i, j, k)_{\mathbf{r}}$.

Next, fix $\Omega=\Omega(i, j, k)_{\mathbf{r}}$ arbitrarily, and write the corresponding $A_{i, j, k}$ as $\left\{s_{1}, s_{2}, s_{3}\right\}$. Consider $(3 n-3)$ FPCR functions

$$
\left\{\chi\left(s_{1}, s_{2}, s_{3}, x\right) \mid x \in\left\{p_{1}, \cdots, p_{2 n}, q_{1}, \cdots, q_{n}\right\}-\left\{s_{1}, s_{2}, s_{3}\right\}\right\},
$$

where we set $q_{\ell}=\sigma\left(g_{\ell}\right)\left(r_{\ell}\right)$ for every $\ell$, and write each $\chi\left(s_{1}, s_{2}, s_{3}, x\right)$ as $\chi_{x}$. Then, since $s_{1}, s_{2}, s_{3}$ are distinct, $\chi_{p_{2 \ell-1}}, \chi_{p_{2 \ell}}, \chi_{r_{\ell}}$, and $\chi_{q_{\ell}}$ determine the equivalence class of the fixed points and the multiplier of $\sigma\left(g_{\ell}\right)$ for every $\ell$ and $\widehat{\sigma} \in \Omega$. (For a more explicit description, see Remark 2.6] below.) Therefore, a point $[\widehat{\sigma}] \in \Omega$ is determined uniquely from the vector

$$
\Lambda_{\Omega}=\left(\widehat{\chi}_{1}, \cdots, \widehat{\chi}_{3 n-3}\right)
$$

at $[\widehat{\sigma}]$, which implies that $\Lambda_{\Omega}$ defined as above gives a holomorphic injection. Thus we have proved the assertion.

Definition 2.5. Such a map $\Lambda_{\Omega_{j}}$, or the set $\left\{\widehat{\chi}_{k}\right\}_{k=1}^{3 n-3}$, as in the proof of Theorem 2.4. is called a global coordinate system of $\Omega_{j}$.

Remark 2.6. If $\sigma\left(g_{\ell}\right)$ is not parabolic, then we can take as a representative of $\left[\sigma\left(g_{\ell}\right)\right]$ the Möbius transformation $g_{\ell}^{\sigma}$ determined by

$$
\frac{g_{\ell}^{\sigma}(z)-\chi_{p_{2 \ell-1}}}{g_{\ell}^{\sigma}(z)-\chi_{p_{2 \ell}}}=\frac{\left(\chi_{r_{\ell}}-\chi_{p_{2 \ell}}\right)\left(\chi_{q_{\ell}}-\chi_{p_{2 \ell-1}}\right)}{\left(\chi_{r_{\ell}}-\chi_{p_{2 \ell-1}}\right)\left(\chi_{q_{\ell}}-\chi_{p_{2 \ell}}\right)} \frac{z-\chi_{p_{2 \ell-1}}}{z-\chi_{p_{2 \ell}}} .
$$


If $\sigma\left(g_{\ell}\right)$ is parabolic, we can take as a representative of $\left[\sigma\left(g_{\ell}\right)\right]$ the Möbius transformation $g_{\ell}^{\sigma}$ determined by

$$
\frac{1}{g_{\ell}^{\sigma}(z)-\chi_{p_{2 \ell}}}=\frac{1}{z-\chi_{p_{2 \ell}}}+\frac{\chi_{r_{\ell}}-\chi_{q_{\ell}}}{\left(\chi_{r_{\ell}}-\chi_{p_{2 \ell}}\right)\left(\chi_{q_{\ell}}-\chi_{p_{2 \ell}}\right)} .
$$

From these equations, we can see that non-parabolic $g_{\ell}^{\sigma}$ tend to parabolic ones continuously. Indeed, the equation (2.1) implies that

$$
\begin{aligned}
& \frac{\chi_{p_{2 \ell-1}}-\chi_{p_{2 \ell}}}{g_{\ell}^{\sigma}(z)-\chi_{p_{2 \ell}}}=\frac{\chi_{p_{2 \ell-1}}-\chi_{p_{2 \ell}}}{z-\chi_{p_{2 \ell}}} \\
& \quad+\frac{\left(\chi_{r_{\ell}}-\chi_{q_{\ell}}\right)\left(\chi_{p_{2 \ell-1}}-\chi_{p_{2 \ell}}\right)}{\left(\chi_{r_{\ell}}-\chi_{p_{2 \ell}}\right)\left(\chi_{q_{\ell}}-\chi_{p_{2 \ell}}\right)}+O\left(\left|\chi_{p_{2 \ell-1}}-\chi_{p_{2 \ell}}\right|^{2}\right)
\end{aligned}
$$

as $\chi_{p_{2 \ell-1}}$ tend to $\chi_{p_{2 \ell}}$.

Finally, we show the following proposition.

Proposition 2.7. For every two $\Omega_{1}, \Omega_{2}$ defined as above, $\Lambda_{\Omega_{1}} \circ \Lambda_{\Omega_{2}}^{-1}$ is a rational function on $\Lambda_{\Omega_{2}}\left(\Omega_{2} \cap \Omega_{1}\right)$. In particular, the coordinate changes between $\Omega_{j}$ are bi-rational.

To prove Proposition 2.7, let $\Omega_{h}=\Omega\left(i_{h}, j_{h}, k_{h}\right)_{\mathbf{r}_{h}}$ for each $h=1,2$. Write the associated $A_{i_{h}, j_{h}, k_{h}}, \mathbf{r}_{h}$, and $\mathbf{q}_{h}$ as $A_{h}=\left\{s_{h, 1}, s_{h, 2}, s_{h, 3}\right\},\left(r_{h, \ell}\right)$, and $\left(q_{h, \ell}\right)$, respectively, for each $h$. Also set

$$
\chi_{x}^{(h)}=\chi\left(s_{h, 1}, s_{h, 2}, s_{h, 3}, x\right)
$$

for every $x \in\left\{p_{1}, \cdots, p_{2 n}, q_{h, 1}, \cdots, q_{h, n}\right\}-\left\{s_{h, 1}, s_{h, 2}, s_{h, 3}\right\}$.

Now, if $\left(r_{1, \ell}\right)=\left(r_{2, \ell}\right)$, then we have the following lemma.

Lemma 2.8. If $\left(r_{1, \ell}\right)=\left(r_{2, \ell}\right)$, then $\left(q_{1, \ell}\right)=\left(q_{2, \ell}\right)$ and we have

$$
\chi_{x}^{(2)}=\frac{\chi_{s_{2,1}}^{(1)}-\chi_{s_{2,2}}^{(1)}}{\chi_{s_{2,1}}^{(1)}-\chi_{s_{2,3}}^{(1)}} \cdot \frac{\chi_{x}^{(1)}-\chi_{s_{2,3}}^{(1)}}{\chi_{x}^{(1)}-\chi_{s_{2,2}}^{(1)}}
$$

for every $x \in\left\{p_{1}, \cdots, p_{2 n}, q_{2,1}, \cdots, q_{2, n}\right\}-\left\{s_{2,1}, s_{2,2}, s_{2,3}\right\}$.

Proof. Recall that $\chi\left(s_{1,1}, s_{1,2}, s_{1,3}, z\right)$ is a Möbius transformation of $z$. Hence, we have that

$$
\chi_{x}^{(2)}=\chi\left(s_{2,1}, s_{2,2}, s_{2,3}, x\right)=\chi\left(\chi_{s_{2,1}}^{(1)}, \chi_{s_{2,2}}^{(1)}, \chi_{s_{2,3}}^{(1)}, \chi_{x}^{(1)}\right),
$$

which is the desired equation.

Next, for general cases, we need the following lemma.

Lemma 2.9. Suppose that $\left(i_{1}, j_{1}, k_{1}\right)=\left(i_{2}, j_{2}, k_{2}\right)$ and that there is only one $\ell_{0}$ such that $r_{1, \ell_{0}} \neq r_{2, \ell_{0}}$. Then we have

$$
\chi_{q_{2}, \ell_{0}}^{(2)}=\frac{\left(\chi_{p_{2 \ell_{0}-1}}^{(1)}+\chi_{p_{2 \ell_{0}}}^{(1)}\right) \chi_{q_{1, \ell_{0}}}^{(1)}-\chi_{p_{2 \ell_{0}-1}}^{(1)} \chi_{p_{2 \ell_{0}}}^{(1)} .}{\chi_{q_{1, \ell_{0}}}^{(1)}} .
$$

Proof. First consider only such $[\widehat{\sigma}] \in \Omega_{1} \bigcap \Omega_{2}$ that $\sigma\left(g_{\ell_{0}}\right)$ are non-parabolic. Also, $\left(i_{1}, j_{1}, k_{1}\right)=\left(i_{2}, j_{2}, k_{2}\right)$ is denoted by $(i, j, k)$. Then $3 n-4$ coordinates $\chi_{x}^{(1)}$ and $\chi_{x}^{(2)}$ are the same, which we write as $\chi_{x}$, except for $\chi_{1}=\chi_{q_{1}, \ell_{0}}^{(1)}$ and $\chi_{2}=\chi_{q_{2}, \ell_{0}}^{(2)}$. Since $\sigma\left(g_{\ell_{0}}\right)$ are non-parabolic, $p_{2 \ell_{0}-1}, p_{2 \ell_{0}}, q_{1, \ell_{0}}$, and $q_{2, \ell_{0}}$ are distinct, and we have

$$
\chi\left(\chi_{p_{2 \ell_{0}-1}}, \chi_{p_{2 \ell_{0}}}, \chi_{r_{1, \ell_{0}}}, \chi_{r_{2, \ell_{0}}}\right)=\chi\left(\chi_{p_{2 \ell_{0}-1}}, \chi_{p_{2 \ell_{0}}}, \chi_{1}, \chi_{2}\right) \text {. }
$$


Here $\chi_{r_{1, \ell_{0}}}$ and $\chi_{r_{2, \ell_{0}}}$ are constants in $\{0,1, \infty\}$, since $r_{h, \ell_{0}} \in A_{1}=A_{2}$. We may assume without loss of generality that $\chi_{r_{1, \ell_{0}}}=0$ and $\chi_{r_{2, \ell_{0}}}=\infty$. Then, we obtain the equation

$$
\frac{1}{\chi_{p_{2 \ell_{0}-1}}}=\frac{\chi_{2}-\chi_{1}}{\left(\chi_{p_{2 \ell_{0}-1}}-\chi_{1}\right)\left(\chi_{2}-\chi_{p_{2 \ell_{0}}}\right)}
$$

which, in turn, implies the desired equality on a generic subset of $\Omega_{1} \cap \Omega_{2}$.

Finally, since $\chi_{q_{2}, \ell_{0}}^{(2)}$ is holomorphic on $\Omega_{2}$, the rational function on the righthand side of (2.2) is continuous and hence has no indefinite singularity on the whole $\Omega_{1} \cap \Omega_{2}$. Thus we conclude the assertion.

Proof of Proposition 2.7. By using the formulas as in Lemmas 2.8 and 2.9, we can conclude the assertion by using the same argument as in the proof of Lemma 2.9.

\section{A neW Distance on the Schottky space}

Recall that a Schottky group is, by definition, a purely loxodromic free Kleinian group having a proper subset of $\widehat{\mathbb{C}}$ as the limit set, which is also characterized as a geometrically finite purely loxodromic free Kleinian group. It is known that Schottky groups are quasi-conformally stable, and hence $S(G)$ is a connected open subset of $\operatorname{EL}(G)$. (See, for instance, [12.) Furthermore, the following fact is fundamental, and is a corollary of a famous theorem of Jørgensen [9].

Proposition 3.1. The closure $\overline{S(G)}$ of the Schottky space $S(G)$ in $X(G)$ is a compact subset of $F D(G)=F(G) \cap D(G)$, which in turn is a proper subset of $\operatorname{EL}(G)$.

Remark 3.2. The interior of $F D(G)$ is $S(G)$. Also the density theorem in [14] implies that $\overline{S(G)}=F D(G)$. (Cf. 6].)

As for the complement of $S(G)$, Minsky shows in [13 that the outer automorphism group $\operatorname{Out}(G)$ acts properly discontinuously on a domain strictly larger than $S(G)$.

Now, we can equip every point $[\sigma]$ in $S(G)$ with the canonical marking $\mathbf{p}=$ $\left(p_{1}, \cdots, p_{2 n}\right)$ by setting $p_{2 j-1}$, and $p_{2 j}$ be the attracting fixed point and the repelling one of $\sigma\left(g_{j}\right)$, respectively, for every $j$. It is clear that, by this canonical marking, we have a holomorphic embedding

$$
\rho: S(G) \rightarrow \widehat{\mathrm{EL}}(G) .
$$

Hence, we consider $S(G)$ as a subset of $\widehat{\operatorname{EL}}(G)$. We write points $[\sigma]$ of $S(G)$ with the canonical marking as $[\widehat{\sigma}]$.

Remark 3.3. The inverse image $\tau^{-1}(S(G))$ consists of $2^{n}$ components, each of which is bi-holomorphic to $S(G)$. While, the closure of $\tau^{-1}(S(G))$ is connected.

Here, we note the following fact.

Lemma 3.4. Every generic set $\Omega=\Omega(i, j, k)_{\mathbf{r}}$ as in the proof of Theorem 2.4 contains $S(G)$ and the corresponding global coordinate system $\Lambda_{\Omega}$ of $\Omega$ gives a holomorphic injection of $S(G)$ into $(\mathbb{C}-\{0,1\})^{3 n-3}$.

Moreover, there is a point of $\overline{S(G)}-S(G)$ where $\Lambda_{\Omega}$ takes a value in $(\mathbb{C}-$ $\{0,1\})^{3 n-3}$. 
Proof. As noted before, every component of $\Sigma_{F P}(\widehat{\sigma})$ is a fixed point of an element of $\sigma(G)$. On the other hand, if $[\widehat{\sigma}] \in S(G)$, then every non-identical element of $\sigma(G)$ has two distinct fixed points, and hence we can see that every two components of $\Sigma_{F P}(\widehat{\sigma})$ are distinct, which implies the first assertion.

Next, the existence of purely loxodromic free Kleinian groups on $\overline{S(G)}-S(G)$ implies the second assertion.

The above proof also implies that every FPCR function is defined on the whole $S(G)$ and takes values in $\mathbb{C}-\{0,1\}$. Hence, we can define distances on $S(G)$ by using FPCR functions.

Definition 3.5. Let $\Lambda$ be a set of FPCR functions, and $d_{h}$ is the hyperbolic distance on $\mathbb{C}-\{0,1\}$. Set

$$
d_{\Lambda}\left(\left[\widehat{\sigma}_{1}\right],\left[\widehat{\sigma}_{2}\right]\right)=\sup _{\widehat{\chi} \in \Lambda} d_{h}\left(\widehat{\chi}\left(\left[\widehat{\sigma}_{1}\right]\right), \widehat{\chi}\left(\left[\widehat{\sigma}_{2}\right]\right)\right)
$$

for every $\left[\widehat{\sigma}_{1}\right],\left[\widehat{\sigma}_{2}\right] \in S(G)$.

If $\Lambda$ is the set of all FPCR functions, then we write $\Lambda$ as $\Lambda_{C R}$ and $d_{\Lambda}$ as $d_{C R}$. We call $d_{C R}$ the FPCR-distance on $S(G)$.

Note that $d_{\Lambda}$ is a distance if $\Lambda$ contains all FPCR functions in some global coordinate system. Also, recall that a classical theorem of Teichmüller (cf. [10]) implies that the FPCR-distance is not greater than the Teichmüller distance on $S(G)$. Furthermore, the proof of Lemma 3.4 implies the following fact.

Corollary 3.6. For every set $\Lambda$ of a finite number of FPCR functions such that $d_{\Lambda}$ is a distance, $d_{\Lambda}$ is not complete on $S(G)$.

Here, we say that a distance $d$ is complete on $S(G)$ if every closed ball

$$
B=\left\{[\widehat{\sigma}] \in S(G) \mid d\left(\left[\widehat{\sigma}_{0}\right],[\widehat{\sigma}]\right) \leq R\right\}
$$

with finite radius $R$ and center $\left[\widehat{\sigma}_{0}\right]$ in $S(G)$ is compact.

Finally, we show the following theorem.

Theorem 3.7. The FPCR-distance $d_{C R}$ is complete on $S(G)$.

Proof. Suppose that there were a non-compact closed ball $B$ with a finite radius $r$ in $S(G)$ with respect to the FPCR-distance. Then, there is a sequence $\left\{\left[\widehat{\sigma_{n}}\right]\right\}$ in $B$ converging to some $\left[\widehat{\sigma_{\infty}}\right]$ in $\widehat{\operatorname{EL}}(G)-S(G)$. Since $G_{\infty}=\sigma_{\infty}(G)$ is a free Kleinian group by Proposition [3.1, either $G_{\infty}$ contains a parabolic element $g^{*}$, or $G_{\infty}$ is purely loxodromic and the limit set of $G_{\infty}$ is $\widehat{\mathbb{C}}$.

In the former case, there are at least two distinct points in

$$
\text { Fix } \sigma_{\infty}\left(g_{1}\right) \bigcup \text { Fix } \sigma_{\infty}\left(g_{2}\right) \bigcup g_{1}\left(\text { Fix } \sigma_{\infty}\left(g_{2}\right)\right)
$$

which are not the single fixed point $s^{*}$ of $g^{*}$. Fix such points and write them as $s_{\infty, 1}, s_{\infty, 2}$. Let $g_{n}^{*}, s_{n, 1}$, and $s_{n, 2}$ be the element in $G_{n}=\sigma_{n}(G)$ and the fixed points corresponding to $g^{*}, s_{\infty, 1}$, and $s_{\infty, 2}$, respectively, for every $n$. Here, letting $\left[\widehat{\sigma_{0}}\right]$ be the center of $B$, we set $G_{0}=\sigma_{0}(G)$. Also, without loss of generality, we may assume that $G_{n}$ converges to $G_{\infty}$ algebraically. Then, since $g_{n}^{*}$ converges to $g^{*}$ in $\mathrm{PSL}_{2}(\mathbb{C})$ and both of the distinct fixed points $s_{n, 1}^{*}$ and $s_{n, 2}^{*}$ of $g_{n}^{*}$ converge to $s^{*}$, the cross ratio $\chi_{n}=\chi\left(s_{n, 1}, s_{n, 2}, s_{n, 1}^{*}, s_{n, 2}^{*}\right) \in \mathbb{C}-\{0,1\}$ tends to

$$
\chi_{\infty}=\chi\left(s_{\infty, 1}, s_{\infty, 2}, s^{*}, s^{*}\right)=0
$$


as $n \rightarrow \infty$. So we can find an $N$ such that

$$
d_{h}\left(\chi_{0}, \chi_{N}\right)>2 r .
$$

On the other hand, since the orbit $G_{0}\left(s_{0}\right)$ of any component $s_{0}$ of $\Sigma_{F P}\left(\widehat{\sigma_{0}}\right)$ (considered as a point) by $G_{0}$ is dense in the limit set $\Lambda\left(G_{0}\right)$ of $G_{0}$, we can find sequences of components $s_{0, j, k}$ of $\Sigma_{F P}\left(\widehat{\sigma_{0}}\right)$ such that

$$
\lim _{k \rightarrow \infty} s_{0, j, k}=s_{0, j}^{*}
$$

for each $j=1,2$. Let $s_{n, j, k}$ be the component of $\Sigma_{F P}\left(\widehat{\sigma_{n}}\right)$ corresponding to $s_{0, j, k}$ for every $n, j$, and $k$. Then, the cross ratio $\chi\left(s_{n, 1}, s_{n, 2}, s_{n, 1, k}, s_{n, 2, k}\right)$ is the value of the same FPCR function, say $\widehat{\chi}_{k}^{*}$, at $\left[\widehat{\sigma}_{n}\right]$ for every $k$ and $n$.

Since $G_{0}$ and $G_{n}$ are quasiconformally conjugate, we can see that

$$
\lim _{k \rightarrow \infty} s_{n, j, k}=s_{n, j}^{*}
$$

and hence

$$
\lim _{k \rightarrow \infty} \widehat{\chi}_{k}^{*}\left(\left[\widehat{\sigma_{n}}\right]\right)=\chi_{n}
$$

for every $n$. Hence, we can find a $K$ such that

$$
d_{h}\left(\widehat{\chi}_{K}^{*}\left(\left[\widehat{\sigma_{0}}\right]\right), \widehat{\chi}_{K}^{*}\left(\left[\widehat{\sigma_{N}}\right]\right)\right)>r
$$

which implies that

$$
d_{C R}\left(\left[\widehat{\sigma}_{0}\right],\left[\widehat{\sigma}_{N}\right]\right)>r .
$$

But this contradicts the assumption that $\left[\widehat{\sigma}_{N}\right] \in B$, which shows the assertion in this case.

Next, in the latter case, $G_{\infty}$ is purely loxodromic and geometrically infinite. Here, we recall the following characterization of geometrically infinite ends by Bonahon [2].

Lemma 3.8 (Cf. [3, Proposition 6.2]). Let $N$ be a hyperbolic 3-manifold with finitely generated fundamental group, and let $E$ be an end of the complement of $\epsilon$-thin cusps of $N$ (with sufficiently small $\epsilon>0$ ). Then $E$ is not geometrically finite if and only if there exists a sequence of closed geodesics exiting $E$.

Proof of Theorem 3.7, continued. In our case, the above characterization implies that there is a sequence of disjoint closed geodesics $\gamma_{m}$ in the hyperbolic manifold $N_{\infty}=\mathbb{H}^{3} / G_{\infty}$ such that, for every compact set $A$ in $N_{\infty}$, there are only a finite number of $\gamma_{m}$ which intersect $A$. In other words, for every sequence $\left\{g_{m}^{*}\right\}$ of elements $g_{m}^{*} \in G_{\infty}$ whose axes project to $\gamma_{m}$, we have that

$$
\chi_{\infty}\left(g_{m}^{*}\right)=\chi\left(s_{\infty, 1}, s_{\infty, 2}, q_{m, 1}^{*}, q_{m, 2}^{*}\right) \rightarrow 0
$$

as $m \rightarrow \infty$, where $s_{\infty, 1}, s_{\infty, 2}$ and $q_{m, 1}^{*}, q_{m, 2}^{*}$ are the two fixed points of $g_{1}^{\infty}=\sigma_{\infty}\left(g_{1}\right)$ and those of $g_{m}^{*}$, respectively, for every $m>1$.

On the other hand, since $G_{0}$ is geometrically finite, i.e., the convex core of $G_{0}$ is compact in $\mathbb{H}^{3} / G_{0}$, we can find a sequence of elements $g_{m}^{0} \in G_{0}$ which corresponds to a sequence $\left\{g_{m}^{*, 0}\right\}$ in $G_{\infty}$ such that the axis of $g_{m}^{*, 0}$ projects to $\gamma_{m}$ for every $m$ and satisfies that the hyperbolic distance between the axis of $g_{1}^{0}=\sigma_{0}\left(g_{1}\right)$ and that of $g_{m}^{0}$ is bounded for all $m$. Namely, there is a constant $\eta>0$ such that

$$
\chi_{0}\left(g_{m}^{0}\right)=\chi\left(s_{0,1}, s_{0,2}, q_{m, 1}^{0}, q_{m, 2}^{0}\right) \geq \eta
$$


for every $m$, where $s_{0,1}, s_{0,2}$ and $q_{m, 1}^{0}, q_{m, 2}^{0}$ are the fixed points of $g_{1}^{0}$ and those of $g_{m}^{0}$. Fix such a sequence $\left\{g_{m}^{0}\right\}$ and hence also $\left\{g_{m}^{*, 0}\right\}$. Then, since $\chi_{\infty}\left(g_{m}^{*, 0}\right)$ tends to 0 , we can find an $M$ such that

$$
d_{h}\left(\chi_{0}\left(g_{M}^{0}\right), \chi_{\infty}\left(g_{M}^{*, 0}\right)\right)>3 r .
$$

Now, we can define

$$
\chi_{n}\left(g_{M}^{n}\right)=\chi\left(s_{n, 1}, s_{n, 2}, q_{M, 1}^{n}, q_{M, 2}^{n}\right)
$$

corresponding to $\chi_{0}\left(g_{M}^{0}\right)$ by using the fixed points $s_{n, 1}, s_{n, 2}$ and $q_{M, 1}^{n}, q_{M, 2}^{n}$ of $g_{1}^{n}$ and $g_{M}^{n}$ in $G_{n}$ which correspond to $g_{1}^{0}$ and $g_{M}^{0}$, respectively, for every $n$. Then, since $G_{n}$ converges to $G_{\infty}$ algebraically,

$$
\lim _{n \rightarrow \infty} \chi_{n}\left(g_{M}^{n}\right)=\chi_{\infty}\left(g_{M}^{*, 0}\right)
$$

and hence we can find an $N$ such that

$$
d_{h}\left(\chi_{0}\left(g_{M}^{0}\right), \chi_{N}\left(g_{M}^{N}\right)\right)>2 r .
$$

Finally, by using the same argument as in the former case, we can find a FPCR function, say $\widehat{\chi}$, such that

$$
d_{h}\left(\widehat{\chi}\left(\left[\widehat{\sigma_{0}}\right]\right), \widehat{\chi}\left(\left[\widehat{\sigma_{N}}\right]\right)>r .\right.
$$

Thus, again we have a contradiction, which shows the assertion.

Remark 3.9. On the Schottky space $S(G)$, every FPCR function is a rational function of $3 n-3$ coordinates in a fixed global coordinate system, which can be shown similarly as in the proof of Proposition 2.7

Also note that the proof of Theorem 3.7 shows that the set $\Lambda$ of all FPCR functions obtained from a given single marked orbit $M O\left(p_{m}\right)$ gives a complete distance $d_{\Lambda}\left(\leq d_{C R}\right)$.

\section{REFERENCES}

[1] Lars V. Ahlfors, Lectures on quasiconformal mappings, 2nd ed., University Lecture Series, vol. 38, American Mathematical Society, Providence, RI, 2006. With supplemental chapters by C. J. Earle, I. Kra, M. Shishikura and J. H. Hubbard. MR2241787 (2009d:30001)

[2] Francis Bonahon, Bouts des variétés hyperboliques de dimension 3, Ann. of Math. (2) 124 (1986), no. 1, 71-158, DOI 10.2307/1971388 (French). MR847953 (88c:57013)

[3] Richard D. Canary, Ends of hyperbolic 3-manifolds, J. Amer. Math. Soc. 6 (1993), no. 1, 1-35, DOI 10.2307/2152793. MR1166330(93e:57019)

[4] Michael Heusener and Joan Porti, The variety of characters in $\mathrm{PSL}_{2}(\mathbb{C})$, Bol. Soc. Mat. Mexicana (3) 10 (2004), 221-237. Special Issue. MR2199350 (2006m:57020)

[5] Rubén A. Hidalgo, Fixed point parameters for Möbius groups, Proyecciones 19 (2000), no. 2, 157-196, DOI 10.4067/S0716-09172000000200005. MR1778800 (2001g:30031)

[6] Rubén Hidalgo and Bernard Maskit, On neoclassical Schottky groups, Trans. Amer. Math. Soc. 358 (2006), no. 11, 4765-4792, DOI 10.1090/S0002-9947-05-03792-X. MR2231871 (2007c:30046)

[7] John Hamal Hubbard, Teichmüller theory and applications to geometry, topology, and dynamics. Vol. 1, Matrix Editions, Ithaca, NY, 2006. Teichmüller theory; With contributions by Adrien Douady, William Dunbar, Roland Roeder, Sylvain Bonnot, David Brown, Allen Hatcher, Chris Hruska and Sudeb Mitra; With forewords by William Thurston and Clifford Earle. MR 2245223 (2008k:30055)

[8] Yoichi Imayoshi and Masahiko Taniguchi, An introduction to Teichmüller spaces, SpringerVerlag, Tokyo, 1992. Translated and revised from the Japanese by the authors. MR.1215481 (94b:32031)

[9] Troels Jørgensen, On discrete groups of Möbius transformations, Amer. J. Math. 98 (1976), no. 3, 739-749. MR0427627 (55 \#658) 
[10] Irwin Kra, On Teichmüller's theorem on the quasi-invariance of cross ratios, Israel J. Math. 30 (1978), no. 1-2, 152-158, DOI 10.1007/BF02760836. MR508260 (82a:30026)

[11] Bernard Maskit, Kleinian groups, Grundlehren der Mathematischen Wissenschaften [Fundamental Principles of Mathematical Sciences], vol. 287, Springer-Verlag, Berlin, 1988. MR.959135 (90a:30132)

[12] Katsuhiko Matsuzaki and Masahiko Taniguchi, Hyperbolic manifolds and Kleinian groups, Oxford Mathematical Monographs, The Clarendon Press Oxford University Press, New York, 1998, Oxford Science Publications. MR 1638795 (99g:30055)

[13] Yair N. Minsky, On dynamics of Out $\left(F_{n}\right)$ on $\mathrm{PSL}_{2}(\mathbb{C})$ characters, Israel J. Math. 193 (2013), no. 1, 47-70, DOI 10.1007/s11856-012-0086-0. MR3038545

[14] Hossein Namazi and Juan Souto, Non-realizability and ending laminations: proof of the density conjecture, Acta Math. 209 (2012), no. 2, 323-395, DOI 10.1007/s11511-012-0088-0. MR3001608

[15] Hiroki Sato, Introduction of new coordinates to the Schottky space. The general case, J. Math. Soc. Japan 35 (1983), no. 1, 23-35, DOI 10.2969/jmsj/03510023. MR679071 (85a:32031)

Department of Information and Computer Sciences, Graduate School of Humanities AND Sciences, NARA Women's University, NARA 630-8506, JAPAN

E-mail address: kimiyo520@gmail.com

Department of Mathematics, Graduate School of Humanities and Sciences, Nara Women's University, NARA 630-8506, JAPAN

E-mail address: tanig@cc.nara-wu.ac.jp 